\title{
Expression Profiles of Exosomal MicroRNAs Derived from Cerebrospinal Fluid in Patients with Congenital Hydrocephalus Determined by MicroRNA Sequencing
}

\author{
Shiyu Chen $\mathbb{D}^{1}{ }^{1}$ Hao Li, ${ }^{2}$ Jicui Zheng, ${ }^{2}$ Lili Hao, ${ }^{3}$ Tianrui Jing, ${ }^{3}$ Peixuan $W u,{ }^{3}$ Bowen Zhang, ${ }^{3}$ \\ Duan Ma $\mathbb{D}^{1,2,3}$ Jing Zhang $\mathbb{D}^{4}$, and Jing Ma ${ }^{4}{ }^{4}$ \\ ${ }^{1}$ Institutes of Biomedical Sciences, Fudan University, Shanghai, China \\ ${ }^{2}$ Children's Hospital of Fudan University, Shanghai, China \\ ${ }^{3}$ School of Basic Medical Sciences, Fudan University, Shanghai, China \\ ${ }^{4}$ ENT Institute, Department of Facial Plastic and Reconstructive Surgery, Eye \& ENT Hospital, Fudan University, Shanghai, China
}

Correspondence should be addressed to Duan Ma; duanma@fudan.edu.cn, Jing Zhang; 13816653516@139.com, and Jing Ma; 14111010077@fudan.edu.cn

Received 6 October 2021; Revised 7 January 2022; Accepted 11 January 2022; Published 4 March 2022

Academic Editor: Anja Hviid Simonsen

Copyright (c) 2022 Shiyu Chen et al. This is an open access article distributed under the Creative Commons Attribution License, which permits unrestricted use, distribution, and reproduction in any medium, provided the original work is properly cited.

Purpose. Congenital hydrocephalus is one of the most common birth defects worldwide. Exosomal microRNAs (miRNAs) in body fluids have been implicated in many diseases. However, their involvement in cerebrospinal fluid from congenital hydrocephalus is not well understood. This study is aimed at investigating the role of dysregulated exosomal miRNAs in congenital hydrocephalus. Methods. We collected cerebrospinal fluid samples from 15 congenital hydrocephalus patients and 21 control subjects. We used miRNA sequencing to generate exosomal miRNA expression profiles in three pairs of samples. We identified 31 differentially expressed exosomal miRNAs in congenital hydrocephalus and predicted their target mRNAs. Results. Three microRNAs (hsamiR-130b-3p, hsa-miR-501-5p, and hsa-miR-2113) were selected according to their fold changes and the function of their target mRNAs, and only hsa-miR-130b-3p and hsa-miR-501-5p were confirmed their expression levels in all samples. Moreover, upregulated hsa-miR-130b-3p might mediate the downregulation of the phosphatase and tensin homolog gene (PTEN), which has been associated with hydrocephalus, via binding to its $3^{\prime}$-untranslated region by dual-luciferase reporter assay. Conclusion. This study implicates that abnormally expressed exosomal miRNAs in cerebrospinal fluid may be involved in the pathomechanism of congenital hydrocephalus.

\section{Introduction}

Congenital hydrocephalus $(\mathrm{CH})$ is among the top five birth defects worldwide, with a prevalence of 4.65 in 10,000 births [1]. As one of the most common congenital central nervous system anomalies, $\mathrm{CH}$ results from the accumulation of cerebrospinal fluid in the brain ventricles, leading to severe neurological damage. The main clinical manifestations are ventriculomegaly, increased intracranial pressure, and brain dysfunction, which may lead to dysgnosia, while ventriculomegaly can critically impair the developmental processes affecting various anatomical and functional aspects of brain maturation [2]. Ventriculoperitoneal shunts are common treatments for $\mathrm{CH}$; however, intracranial pressure may be normal or even low in some patients, including those with other structural brain abnormalities, and surgical shunting may fail to improve the neurological impairment in those patients $[2,3]$. Furthermore, there is a high risk of postoperative complications, such as intracranial infection. It is therefore important to explore the etiology and pathogenesis of $\mathrm{CH}$ to improve its prevention and treatment.

The underlying causes of $\mathrm{CH}$ are currently not well understood, but it is generally believed to be a multifactorial disease involving genetic and environmental interactions. $\mathrm{CH}$ may be associated with chromosomal (e.g., abnormality of chromosome $6 \mathrm{q}$ and Xp22.33) or single gene 
TABLE 1: Basic information of samples for miRNA sequencing.

\begin{tabular}{lcccc}
\hline Patient ID & Age & Sex & Samples & Phenotype \\
\hline CH1 & 15 months & Female & $15 \mathrm{ml}$ cerebrospinal fluid & Congenital hydrocephalus \\
CH2 & 2 months & Male & $15 \mathrm{ml}$ cerebrospinal fluid & Congenital hydrocephalus \\
CH3 & 13 months & Male & $15 \mathrm{ml}$ cerebrospinal fluid & Congenital hydrocephalus \\
CS1 & 14 months & Male & $15 \mathrm{ml}$ cerebrospinal fluid & Intracranial space-occupying lesions \\
CS2 & 13 months & Female & $13 \mathrm{ml}$ cerebrospinal fluid & Tethered cord syndrome \\
CS3 & 10 months & Male & $15 \mathrm{ml}$ cerebrospinal fluid & Intracranial space-occupying lesions \\
\hline
\end{tabular}

$\mathrm{CH}$ : congenital hydrocephalus; CS: control subjects.

TABLE 2: Basic information of $\mathrm{CH}$ patients.

\begin{tabular}{|c|c|c|c|c|}
\hline Patient ID & Age & Sex & Samples & Phenotype \\
\hline $\mathrm{CH} 1$ & 15 months & Female & $15 \mathrm{ml}$ cerebrospinal fluid & Hydrocephalus \\
\hline $\mathrm{CH} 2$ & 2 months & Female & $15 \mathrm{ml}$ cerebrospinal fluid & Hydrocephalus \\
\hline $\mathrm{CH} 3$ & 14 months & Male & $15 \mathrm{ml}$ cerebrospinal fluid & Hydrocephalus \\
\hline $\mathrm{CH} 4$ & 9 months & Female & $7 \mathrm{ml}$ cerebrospinal fluid & Hydrocephalus \\
\hline $\mathrm{CH} 5$ & 29 days & Male & $7 \mathrm{ml}$ cerebrospinal fluid & Hydrocephalus \\
\hline $\mathrm{CH} 6$ & 5 months & Male & $15 \mathrm{ml}$ cerebrospinal fluid & Hydrocephalus \\
\hline $\mathrm{CH} 7$ & 6 months & Male & $7 \mathrm{ml}$ cerebrospinal fluid & Hydrocephalus \\
\hline $\mathrm{CH} 8$ & 4 months & Female & $8 \mathrm{ml}$ cerebrospinal fluid & Hydrocephalus \\
\hline $\mathrm{CH} 9$ & 24 months & Male & $15 \mathrm{ml}$ cerebrospinal fluid & Hydrocephalus \\
\hline $\mathrm{CH} 10$ & 9 months & Female & $8 \mathrm{ml}$ cerebrospinal fluid & Hydrocephalus \\
\hline $\mathrm{CH} 11$ & 24 months & Female & $15 \mathrm{ml}$ cerebrospinal fluid & Hydrocephalus \\
\hline $\mathrm{CH} 12$ & 60 months & Male & $25 \mathrm{ml}$ cerebrospinal fluid & Hydrocephalus \\
\hline $\mathrm{CH} 13$ & 15 months & Female & $11 \mathrm{ml}$ cerebrospinal fluid & Hydrocephalus \\
\hline $\mathrm{CH} 14$ & 14 months & Male & $9 \mathrm{ml}$ cerebrospinal fluid & Hydrocephalus \\
\hline $\mathrm{CH} 15$ & 2 months & Male & $9 \mathrm{ml}$ cerebrospinal fluid & Hydrocephalus \\
\hline
\end{tabular}

$\mathrm{CH}$ : congenital hydrocephalus.

abnormalities [1], and mutations of at least 43 genes/loci have been associated with $\mathrm{CH}$ in animal models or patients [4]. In addition, gene expression disorders may also be involved in the etiology of $\mathrm{CH}$ [5]. Among the factors regulating gene expression, microRNAs (miRNAs; miR) are responsible for modulating nearly one-third of genes and thus regulating a variety of physiological processes [6]. Although miRNAs are found widely both inside and outside cells, about $70 \%$ of all miRNAs are stably distributed in exosomes, instead of in their host cells [7]. Exosomes are phospholipid bilayer vesicles, with a diameter of $40-100 \mathrm{~nm}$. They are secreted by cells and form a cell-to-cell information transmission system by carrying various biological molecules (protein, DNA, mRNA, and miRNA), which in turn affect the physiological state of the cells and are closely related to the occurrence and process of a variety of diseases [8]. Exosomes can cross the blood-brain barrier and are readily accessible in various human biofluids, including cerebrospinal fluid $[9,10]$. The characteristics of exosomes with a substantial miRNA content ensure to act as promising biomarkers in some diseases. For instance, exosomal miR-181$5 \mathrm{p}$ from blood samples was identified as a potential diagnostic biomarker for early-stage non-small-cell lung cancer [8], and exosomal miR-150 and miR-21 from blood samples were indicated as biomarkers for the early detection of colo- rectal cancer [11]. These exosomal miRNAs can be delivered to recipient cells to exert their functions $[12,13]$ by affecting the translation or stability of their target mRNAs via direct interactions [9]. The existence and importance of exosomal miRNAs in cerebrospinal fluid have been confirmed in several neurological disorders, such as Alzheimer's disease [14], Parkinson's disease [15], and intraventricular hemorrhage (IVH) in preterm infants [16]. However, the role of exosomal miRNAs in the cerebrospinal fluid in $\mathrm{CH}$ remains unknown.

In the current study, we characterized the exosomal miRNA profile of cerebrospinal fluid from patients with $\mathrm{CH}$ using miRNA sequencing and bioinformatics analysis and focused on three miRNAs (miR-130b-3p, miR-501-5p, and miR-2113). We verified the differential expression of the above three miRNAs in patients with $\mathrm{CH}$, and we also investigated the expression of phosphatase and tensin homolog gene (PTEN), which was associated with hydrocephalus in previous reports [17] via the mechanism of these miRNAs.

\section{Materials and Methods}

2.1. Patients and Samples. Cerebrospinal fluid samples were obtained from the age-sex-matched $15 \mathrm{CH}$ patients and 21 
TABLE 3: Basic information of CS.

\begin{tabular}{|c|c|c|c|c|}
\hline Patient ID & Age & Sex & Samples & Phenotype \\
\hline CS1 & 15 months & Male & $15 \mathrm{ml}$ cerebrospinal fluid & Intracranial space-occupying lesions \\
\hline CS2 & 12 months & Female & $13 \mathrm{ml}$ cerebrospinal fluid & Congenital tethered cord syndrome \\
\hline CS3 & 17 months & Male & $15 \mathrm{ml}$ cerebrospinal fluid & Intracranial space-occupying lesions \\
\hline CS4 & 5 months & Male & $8 \mathrm{ml}$ cerebrospinal fluid & Congenital tethered cord syndrome \\
\hline CS5 & 14 months & Male & $15 \mathrm{ml}$ cerebrospinal fluid & Intracranial space-occupying lesions \\
\hline CS6 & 22 months & Female & $15 \mathrm{ml}$ cerebrospinal fluid & Congenital tethered cord syndrome \\
\hline CS7 & 6 days & Male & $15 \mathrm{ml}$ cerebrospinal fluid & Intracranial hemorrhage \\
\hline CS8 & 4 months & Male & $10 \mathrm{ml}$ cerebrospinal fluid & Congenital tethered cord syndrome \\
\hline CS9 & 108 months & Female & $10 \mathrm{ml}$ cerebrospinal fluid & Intracranial hemorrhage \\
\hline CS10 & 24 months & Male & $20 \mathrm{ml}$ cerebrospinal fluid & Congenital tethered cord syndrome \\
\hline CS11 & 36 months & Male & $8 \mathrm{ml}$ cerebrospinal fluid & Intracranial space-occupying lesions \\
\hline CS12 & 48 months & Male & $7 \mathrm{ml}$ cerebrospinal fluid & Congenital tethered cord syndrome \\
\hline CS13 & 21 months & Female & $20 \mathrm{ml}$ cerebrospinal fluid & Intracranial hemorrhage \\
\hline CS14 & 9 days & Male & $5 \mathrm{ml}$ cerebrospinal fluid & Congenital tethered cord syndrome \\
\hline CS15 & 72 months & Female & $12 \mathrm{ml}$ cerebrospinal fluid & Congenital tethered cord syndrome \\
\hline CS16 & 12 months & Female & $10 \mathrm{ml}$ cerebrospinal fluid & Intracranial hemorrhage \\
\hline CS17 & 2 months & Male & $6 \mathrm{ml}$ cerebrospinal fluid & Congenital tethered cord syndrome \\
\hline CS18 & 60 months & Female & $10 \mathrm{ml}$ cerebrospinal fluid & Congenital tethered cord syndrome \\
\hline CS19 & 20 days & Male & $20 \mathrm{ml}$ cerebrospinal fluid & Congenital tethered cord syndrome \\
\hline CS20 & 23 months & Female & $15 \mathrm{ml}$ cerebrospinal fluid & Intracranial space-occupying lesions \\
\hline CS21 & 30 months & Female & $6 \mathrm{ml}$ cerebrospinal fluid & Intracranial space-occupying lesions \\
\hline
\end{tabular}

CS: control subjects.

control subjects (CS) from the Children's Hospital of Fudan University. The diagnosis of $\mathrm{CH}$ was carried out at the Children's Hospital of Fudan University and excluded trauma, tumor, secondary hydrocephalus, and infection. The CS were the patients diagnosed with three types of secondary hydrocephalus (intracranial space-occupying lesions, intracranial hemorrhage, and congenital tethered cord syndrome). The cerebrospinal fluid samples were maintained in RNA-free centrifuge tubes after surgery and stored at $-80^{\circ} \mathrm{C}$. miRNA sequencing was carried out using cerebrospinal fluid samples from three $\mathrm{CH}$ patients and three $\mathrm{CS}$ (Table 1). Cerebrospinal fluid samples from $15 \mathrm{CH}$ patients and 21 CS were used for further real-time quantitative qPCR analysis (Tables 2 and 3).

2.2. Exosome Isolation and RNA Extraction. The cerebrospinal fluid samples were centrifuged for $30 \mathrm{~min}$ at $900 \mathrm{~g}$ and $4^{\circ} \mathrm{C}$ to remove cells and large debris. The supernatant was collected and filtered through a $0.22 \mu \mathrm{m}$ filter to remove additional cellular fragments. Exosomes were isolated and purified from the supernatant using an exoEasy Maxi Kit (Qiagen, Hilden, Germany) following the manufacturer's protocol. Total exosomal RNA was extracted using TRIzol reagent (Invitrogen, USA) according to the manufacturer's instructions. The RNA quality and concentration were evaluated based on the optical density 260/280 and 260/230 ratios using a Nano Drop ND-2000 spectrophotometer (Thermo Fisher Scientific, Waltham, MA, USA). RNA integrity was assessed by agarose gel electrophoresis.
2.3. miRNA Sequencing and Data Analysis. Exosomal miRNA libraries were constructed using total RNA samples from exosomes and assessed using an Agilent 2100 Bioanalyzer (Agilent, California, USA). The $3^{\prime}$ and $5^{\prime}$ adapters were ligated to total RNA samples, respectively, and cDNAs were synthesized with the adapter-ligated miRNAs and used as templates for polymerase chain reaction (PCR) amplification. Amplified fragments of 135-155 base pairs were selected to construct miRNA libraries, which were then denatured with $0.1 \mathrm{M} \mathrm{NaOH}$ and sequenced using a TruSeq Rapid SR Cluster Kit (Illumina, CA, USA) with Illumina NextSeq 500, according to the manufacturer's instructions. Raw reads were subjected to quality control to assess the suitability of the raw data for subsequent analysis. Trimmed data were obtained by removing the $3^{\prime}$ adapters and shorter reads ( $\leq 15$ base pairs) from the raw data. The subsequent reads were aligned with the human reference genome annotated with miRNA to generate an miRNA expression value (counts per million reads; CPM) using miRDeep2 [17]. The detected miRNAs were determined based on an average $\mathrm{CPM}$ value $>1$.

2.4. Differential Expression of Exosomal miRNAs. Differentially expressed exosomal miRNAs were identified using edgeR with a threshold fold change $(\mathrm{FC})>1.5$ and $P$ value $\leq 0.05$. We used the CPM value of significantly expressed exosomal miRNAs to perform hierarchical clustering analysis and reveal the expression patterns of the exosomal miRNAs and samples. Scatter plots were generated to assess the 


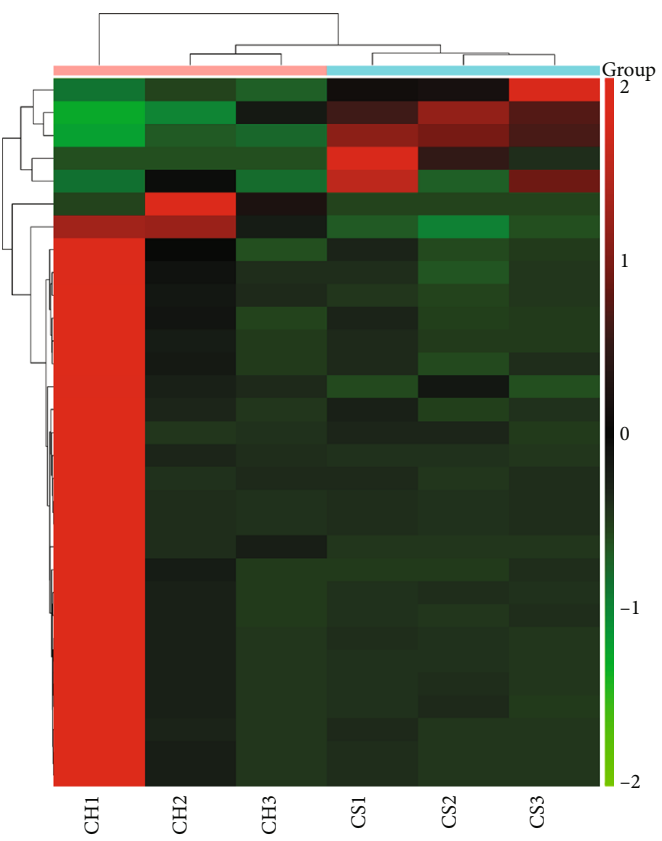

(a)

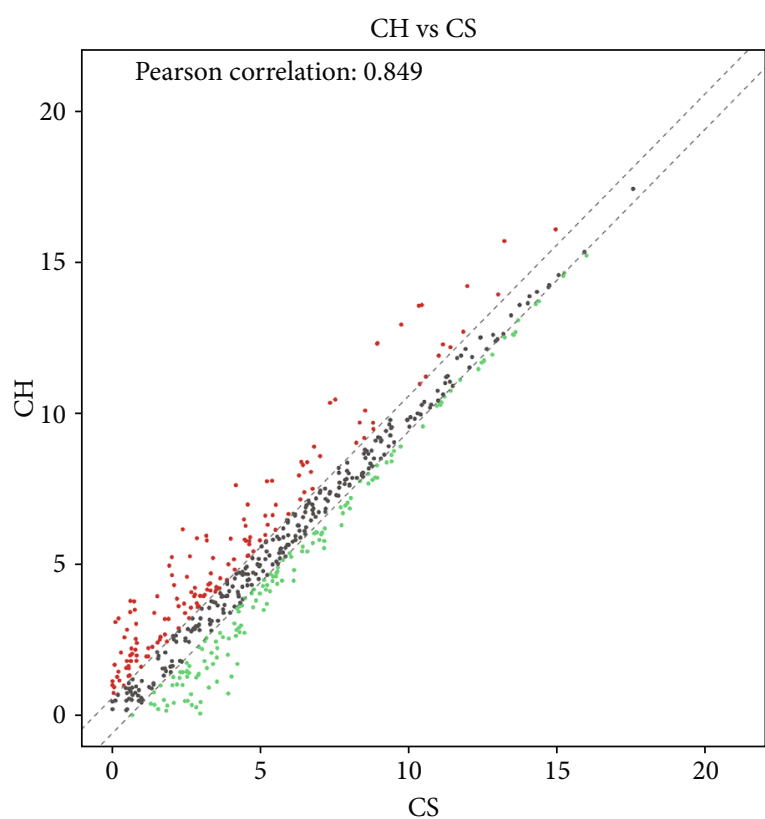

- Up_regulated genes (314)

- Not differential expressed (320)

- Down_regulated genes (276)

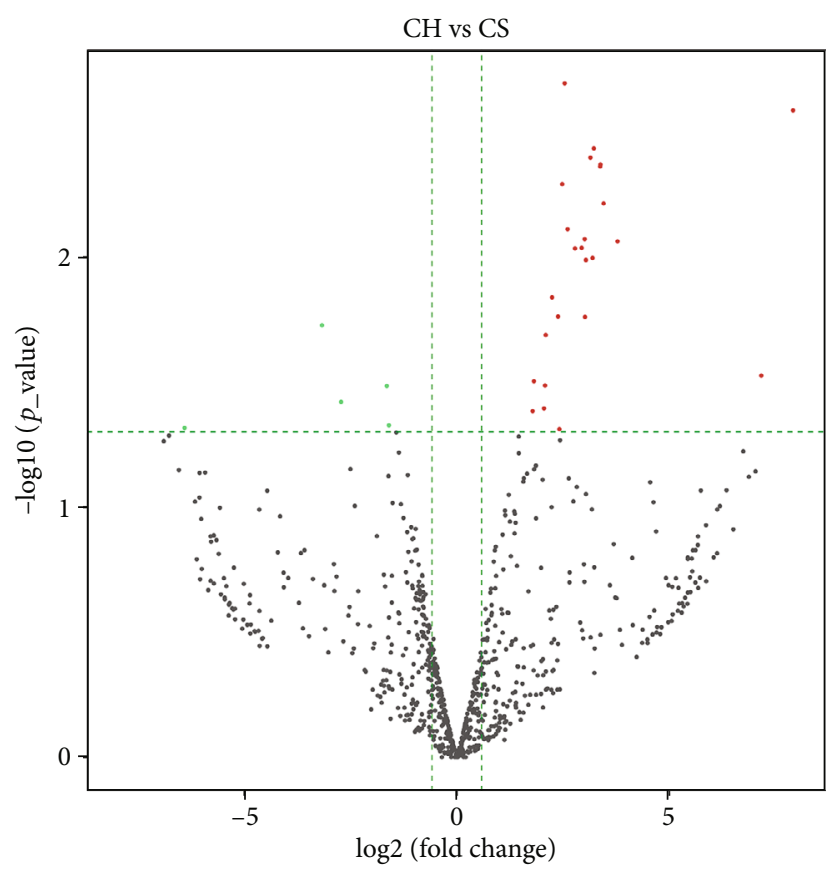

- Up_regulated genes (26)

- Not differential expressed (879)

- Down_regulated genes (5)

(c)

Figure 1: Differential expression profiles of exosomal miRNAs in $\mathrm{CH}$ patients and CS. (a) Hierarchical clustering analysis of exosomal miRNAs between the $\mathrm{CH}$ patients $(\mathrm{CH} 1, \mathrm{CH} 2$, and $\mathrm{CH} 3)$ and $\mathrm{CS}$ (CS1, CS2, and CS3). Expression values are represented by red and green shades, indicating expressions above and below the median expression level across all samples, respectively. (b) The scatter plot of 910 exosomal miRNAs. Pearson's correlation coefficient was 0.849 . The red dots indicate upregulated genes, the green dots indicate downregulated genes, and the black dots indicate nondifferentially expressed genes. (c) The volcano plot of 910 exosomal miRNAs. The fold change threshold is 1.5 and $P$ value $\leq 0.05$. The red dots indicate upregulated genes, the green dots indicate downregulated genes, and the black dots indicate nondifferentially expressed genes. 
TABLE 4: Upregulated and downregulated miRNAs in the volcano plot.

\begin{tabular}{|c|c|c|c|}
\hline \multicolumn{2}{|c|}{ Upregulated miRNAs } & \multicolumn{2}{|c|}{ Downregulated miRNAs } \\
\hline miRNA ID & $\log _{2} \mathrm{FC}$ & miRNA ID & $\log _{2} \mathrm{FC}$ \\
\hline hsa-miR-2113 & 7.910966974 & hsa-miR-501-5p & -6.405186884 \\
\hline hsa-miR-302d-3p & 7.163506356 & hsa-let-7e-3p & -3.172160369 \\
\hline hsa-miR-137-5p & 3.781667006 & hsa-miR-29c-5p & -2.72261609 \\
\hline hsa-miR-320e & 3.453737277 & hsa-miR-223-5p & -1.647330356 \\
\hline hsa-miR-320c & 3.379260772 & hsa-miR-584-5p & -1.59751174 \\
\hline hsa-miR-320c & 3.373467094 & & \\
\hline hsa-miR-320b & 3.223989228 & & \\
\hline hsa-miR-129-5p & 3.194153868 & & \\
\hline hsa-miR-129-5p & 3.194153868 & & c \\
\hline hsa-miR-320b & 3.143871008 & & \\
\hline hsa-miR-130b-3p & 3.037326563 & & \\
\hline hsa-miR-4429 & 3.015750545 & & \\
\hline hsa-miR-320d & 3.008370049 & & \\
\hline hsa-miR-320d & 2.93745457 & & \\
\hline hsa-miR-412-5p & 2.780774639 & & \\
\hline hsa-miR-296-3p & 2.607140495 & & \\
\hline hsa-miR-708-3p & 2.537461519 & & \\
\hline hsa-miR-320a-3p & 2.480146691 & & \\
\hline hsa-miR-1224-5p & 2.413574833 & & \\
\hline hsa-miR-134-5p & 2.381827533 & & \\
\hline hsa-miR-1298-5p & 2.24115725 & & \\
\hline hsa-miR-760 & 2.091967229 & & \\
\hline hsa-miR-136-5p & 2.0757691 & & \\
\hline hsa-miR-181a-3p & 2.051552079 & & \\
\hline hsa-miR-193a-5p & 1.813698773 & & \\
\hline hsa-miR-7704 & 1.784297108 & & \\
\hline
\end{tabular}

FC: fold change.

distribution trends of the miRNAs in the $\mathrm{CS}$ and $\mathrm{CH}$ patients. Differentially expressed exosomal miRNAs were screened based on a $\log _{2} \mathrm{FC}$ and $-\log _{10} P$ value to generate volcano plots demonstrating the relationship between the FC of differential expression and statistical significance.

2.5. The Target Genes of miRNA Prediction, Functional Annotation, and Pathway Enrichment. The miRDB and TargetScan algorithms were used to predict the target genes of exosomal miRNAs that were differentially expressed between the $\mathrm{CS}$ and $\mathrm{CH}$ patients. Functional enrichment of the target genes was then determined by Gene Ontology (GO) (http://www.geneontology.org/) and Kyoto Encyclopedia of Genes and Genomes (KEGG) (http://www.genome.jp/ kegg) analyses.

2.6. Quantitative Polymerase Chain Reaction ( $q P C R$ ). cDNAs were synthesized from $1 \mu \mathrm{g}$ total RNA using a PrimeScript RT Reagent Kit with gDNA Eraser (Takara, Tokyo, Japan), and qPCR was conducted with SYBR Premix Ex Taq $^{\text {TM }}$ (Takara) on a StepOnePlus ${ }^{\text {TM }}$ Real-Time PCR System (Thermo Fisher Scientific). The relative expression levels of
miRNAs and mRNAs were normalized to the housekeeping gene U6 and glyceraldehyde 3-phosphate dehydrogenase (GAPDH), respectively, and were calculated by the relative quantification method $\left(2^{-\Delta \Delta \mathrm{Ct}}\right)$.

The primers used were as follows: hsa-miR-2113-GSP: GGGGATTTGTGCTTGGCTC, hsa-miR-2113-R: GTGC GTGTCGTGGAGTCG; hsa-miR-130b-3p-GSP: GGGCAG TGCAATGATGAAA, hsa-miR-130b-3p-R: GTGCGTGTC GTGGAGTCG; hsa-miR-501-5p-GSP: GGAGAATCCTT TGTCCCTGG, hsa-miR-501-5p-R: GTGCGTGTCGTGGA GTCG; U6-F: GCTTCGGCAGCACATATACTAAAAT, U6-R: CGCTTCACGAATTTGCGTGTCAT; PTEN-F: ACACGACGGGAAGACAAGTT, PTEN-R: CTGGTCCTG GTATGAAGAATG; and GAPDH-F: GGGAAACTGTG GCGTGAT, GAPDH-R: GAGTGGGTGTCGCTGTTGA.

2.7. Cell Culture. Human embryonic kidney 293 (HEK293T) cells were seeded in Dulbecco's modified Eagle's medium (Biological Industries, Kibbutz Beit HaEmek) with 10\% foetal bovine serum (Biological Industries) at $37^{\circ} \mathrm{C}$ in $5 \% \mathrm{CO}_{2}$. All cell culture dishes and culture plates were purchased from Hangzhou Xinyou Biotechnology Co., Ltd.

2.8. Dual-Luciferase Reporter Assay. The recombinant plasmid pGL3-promoter-PTEN-WT (wild-type PTEN $3^{\prime}$ -untranslated region (UTR)) and pGL3-promoter-PTENDel (deleted PTEN $3^{\prime}$-untranslated region (UTR)) were constructed. Mimics and NC oligonucleotides for hsa-miR130b-3p were obtained from RiboBio Co., Ltd. (China). HEK293T cells (Cell Bank, Shanghai, China) were seeded in 96-well plates at $1 \times 10^{4}$ cells per well and incubated overnight at $37^{\circ} \mathrm{C}$. The respective mimics and NC oligonucleotides were cotransfected into HEK293T cells with pGL3promoter-PTEN-WT/pGL3-promoter-PTEN-Del and pGL3-Renilla using Lipofectamine 3000 (Invitrogen). Cells were then harvested $48 \mathrm{~h}$ after transfection. Both firefly and Renilla luciferase activities were measured using a DualLuciferase Reporter Assay System (Promega, USA), and the firefly luciferase activities were normalized to Renilla luciferase activities.

The primers used were as follows: PTEN-3utr-Xba1-F: GCTCTAGAGCtggcaataggacattgtgtc and PTEN-3utr-Xba1R: GCTCTAGAGCgctgccttttctagcaccaatatgc.

2.9. Statistical Analysis. All experiments were repeated three times. All statistical analyses were performed by paired twotailed Student's $t$-tests using GraphPad Software (GraphPad Inc., La Jolla, CA, USA). A value of $P<0.05$ was considered significant.

\section{Results}

3.1. Analysis of Differentially Expressed Exosomal miRNAs in $\mathrm{CH}$ Patients and CS. Differential expression of miRNAs in cerebrospinal fluid exosomes from three $\mathrm{CH}$ patients and three CS was analyzed using edgeR. The criteria for differential miRNA expression were an FC threshold of $1.5, P$ value $\leq 0.05$, and mean $C P M \geq 1 . \log _{2} \mathrm{FC}$ was calculated to represent differential miRNA expression, with a positive value 


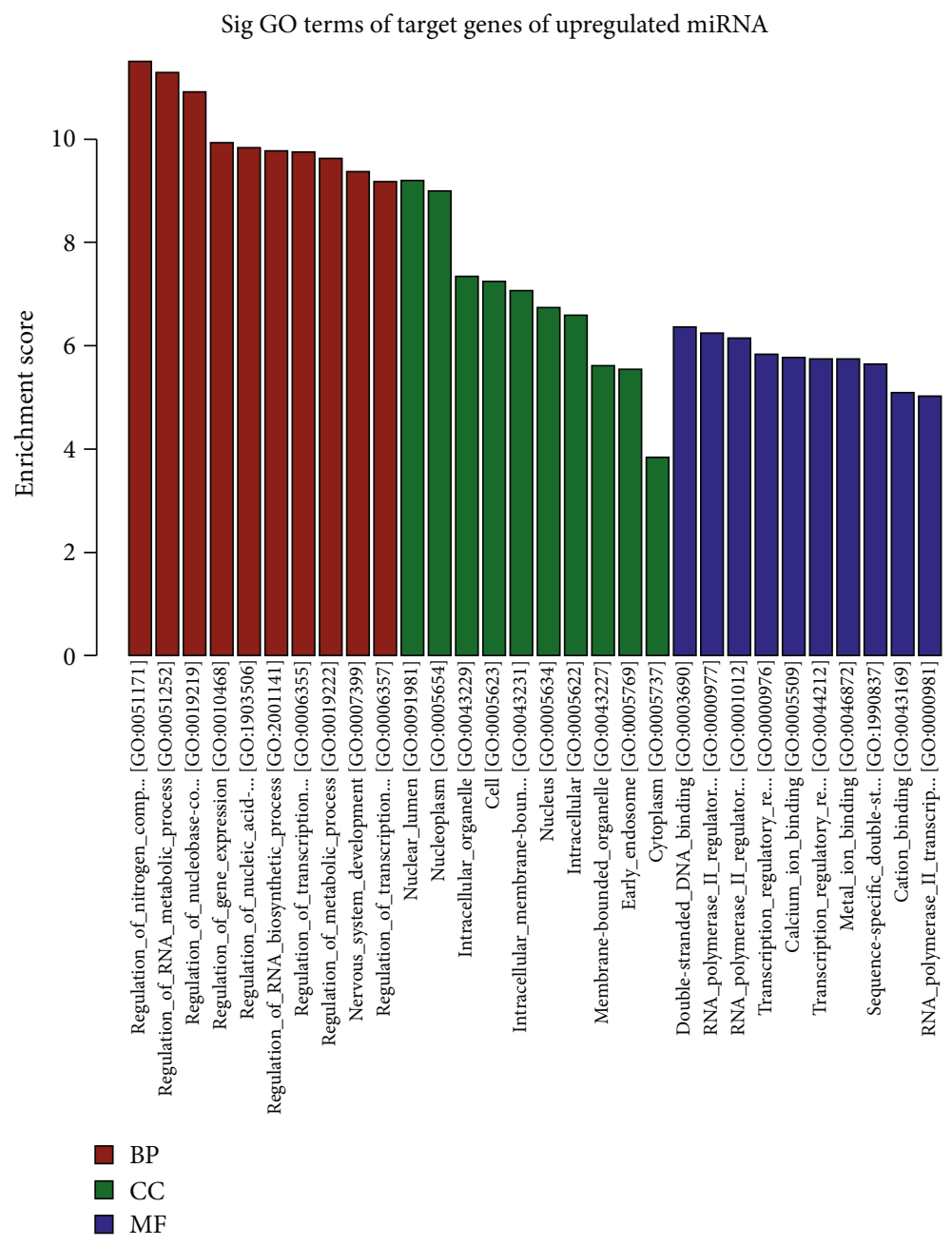

(a)

Figure 2: Continued. 


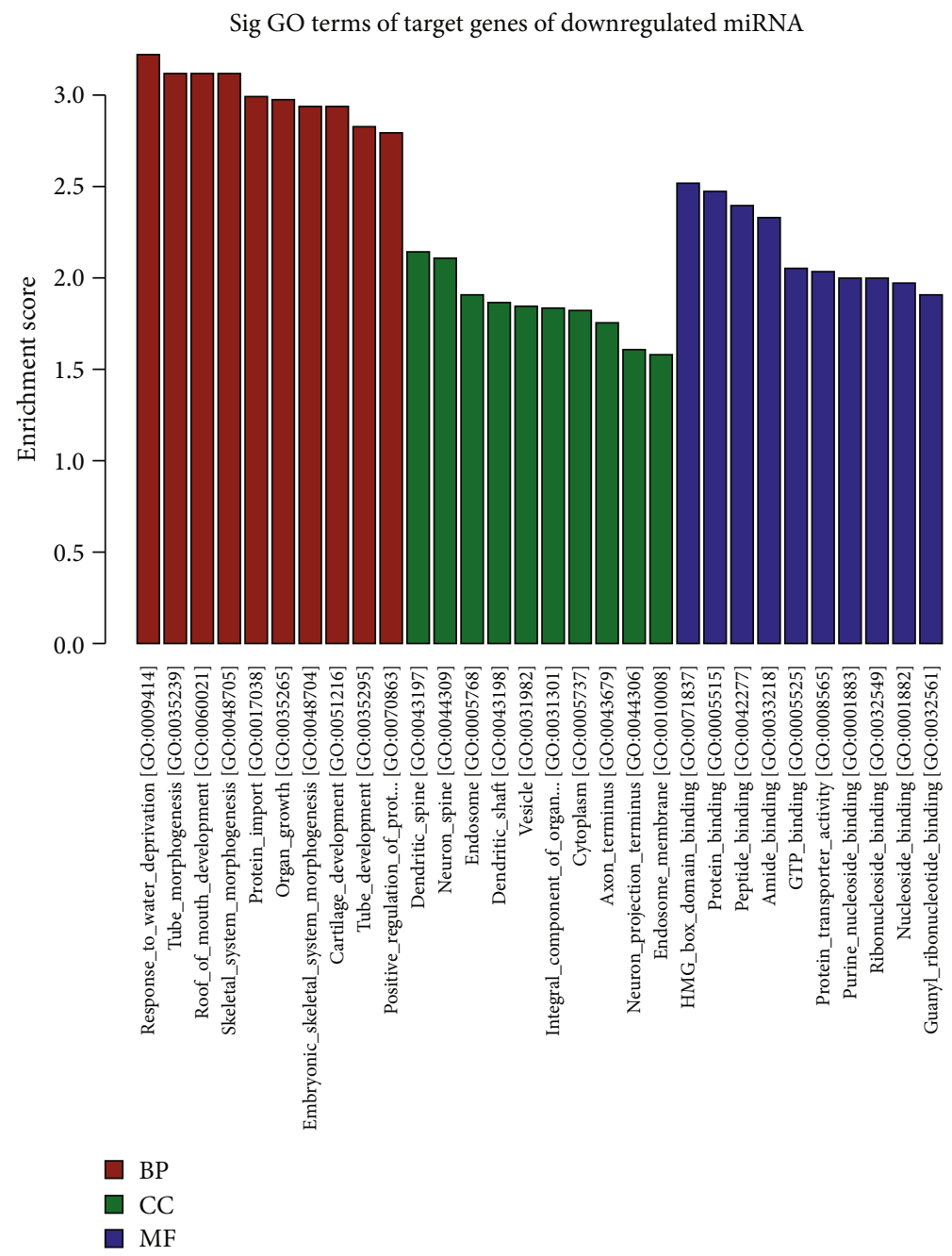

(b)

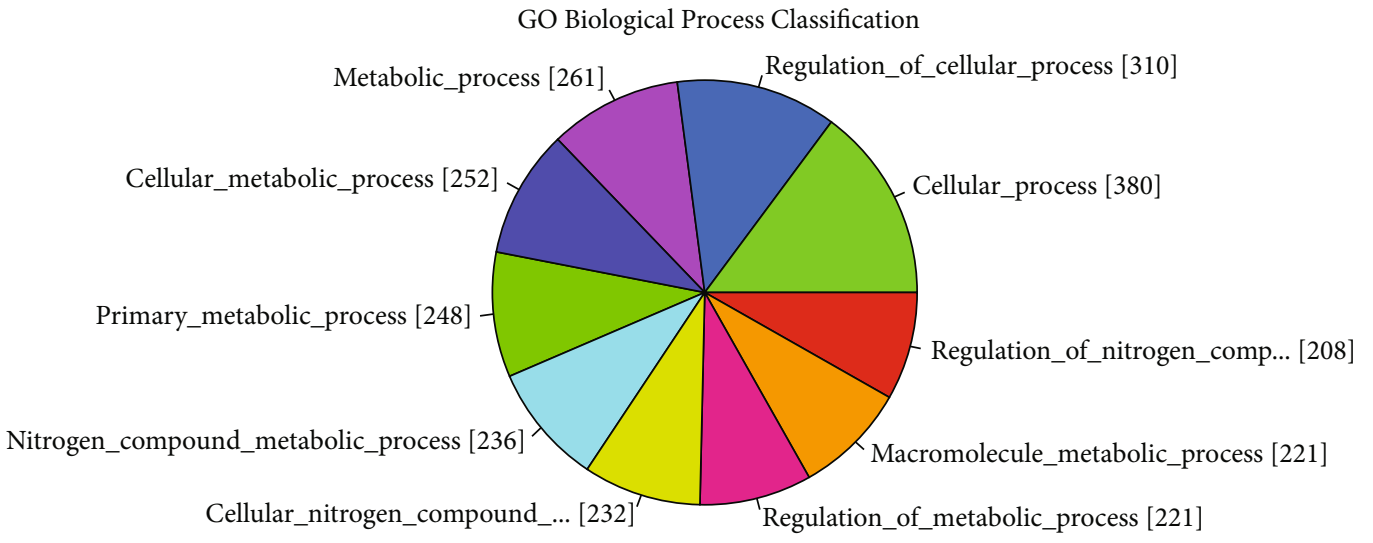

(c)

Figure 2: Continued. 
GO Cellular component Classification

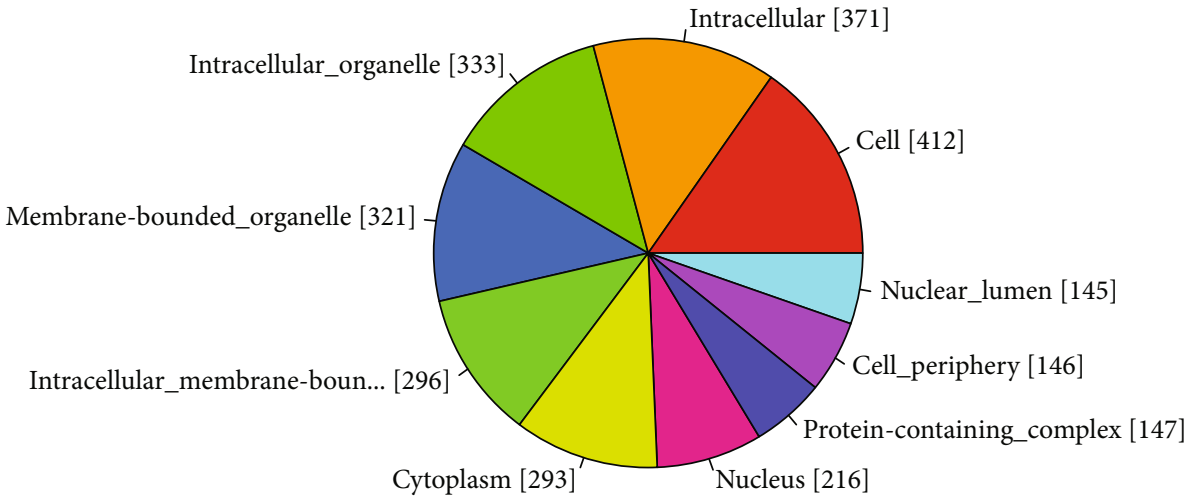

(d)

GO Molecular Function Classification Peptide_binding [309]

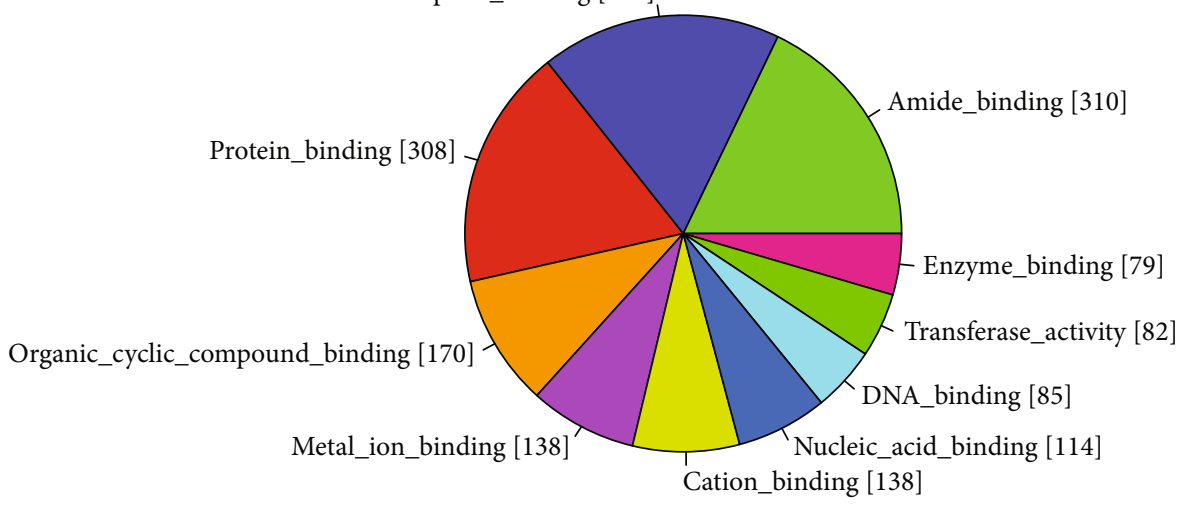

(e)

GO Biological Process Classification

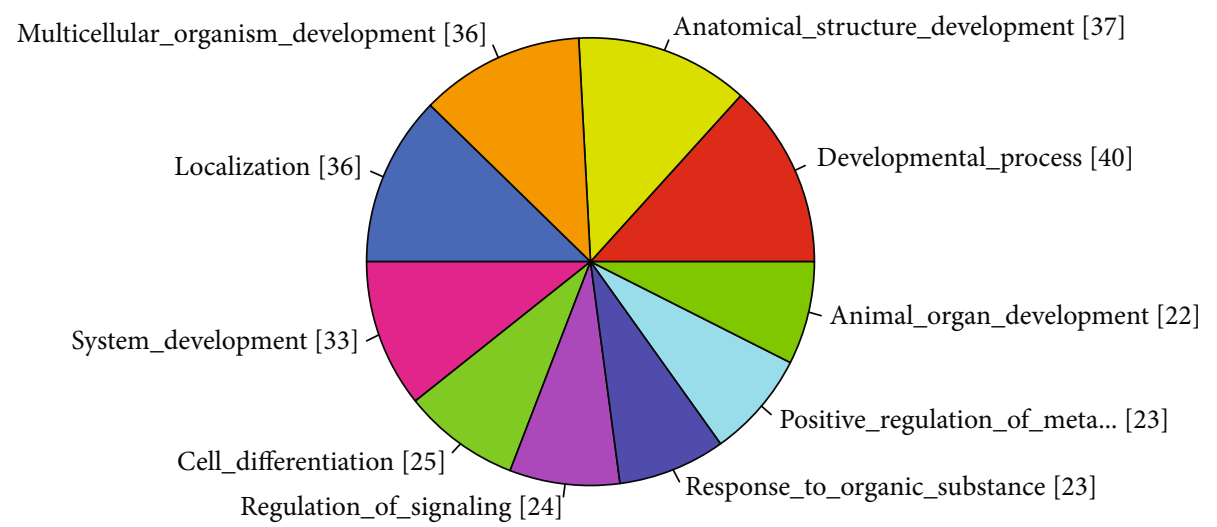

(f)

FIGURE 2: Continued. 
GO Cellular component Classification

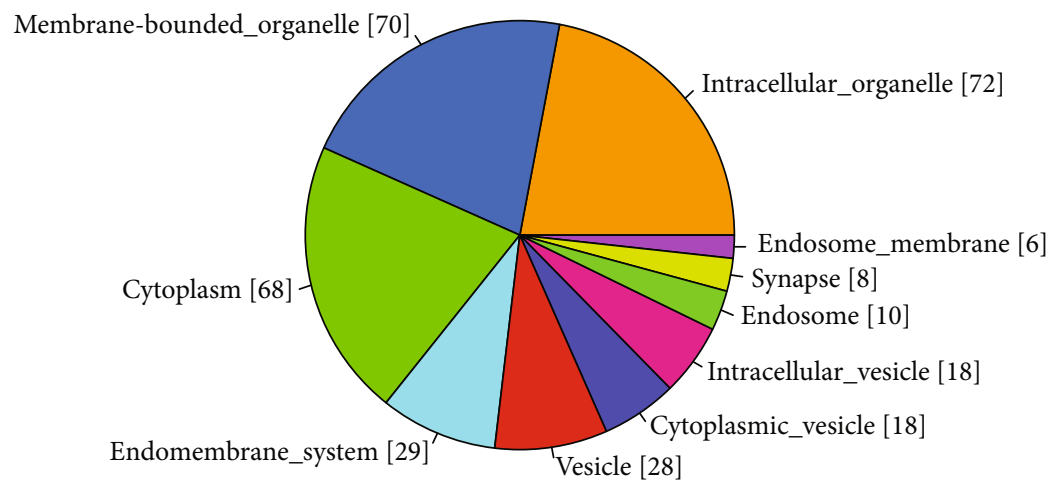

(g)

GO Molecular Function Classification

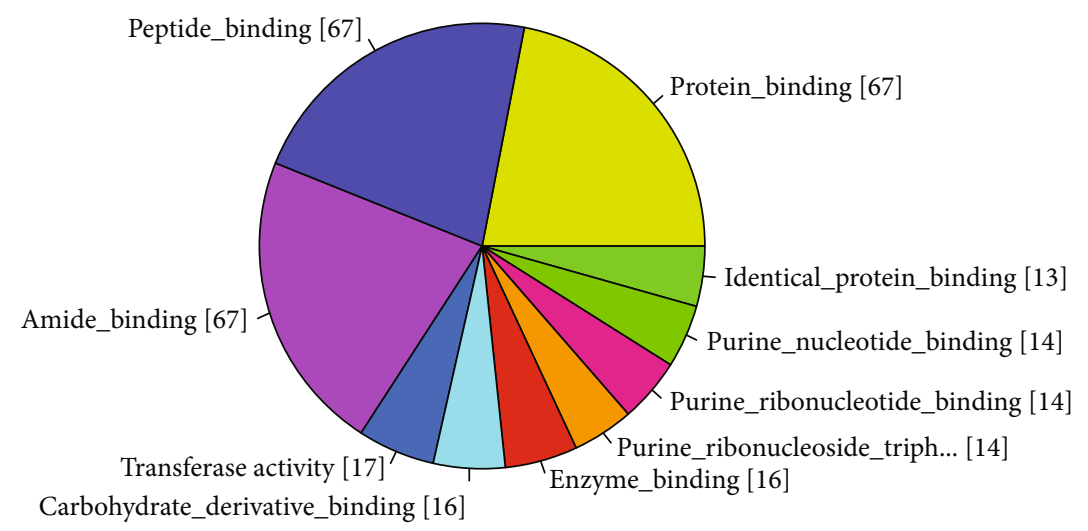

(h)

Figure 2: GO terms analysis of the predicted target genes of differentially expressed exosomal miRNAs in CH patients. (a) Enriched GO terms of differentially upregulated miRNA target genes in $\mathrm{CH}$ patients compared with $\mathrm{CS}$. (b) Enriched GO terms of differentially downregulated miRNA target genes in $\mathrm{CH}$ patients compared with CS. (c, d, e) The top 10 GO terms of biological process, cellular component, and molecular function in differentially upregulated miRNA target genes according to the gene counts included in each term. (f, g, h) The top $10 \mathrm{GO}$ terms of biological process, cellular component, and molecular function in differentially downregulated miRNA target genes according to the gene counts included in each term. BP: biological process; CC: cellular component; MF: molecular function.

indicating upregulation and a negative value indicating downregulation. We identified thousands of differentially expressed human miRNAs.

We performed differential expression analyses of three miRNAs by hierarchical clustering, scatter plots, and volcano plots, respectively. Hierarchical clustering analysis of exosomal miRNA signal intensities revealed evidence of significant differential expression of exosomal miRNAs between the $\mathrm{CH}$ patients and CS (Figure 1(a)). There were 910 miRNAs in the scatter plot, of which 314 and 274 were upregulated and downregulated, respectively (Figure 1(b)). Pearson's correlation coefficient was 0.849. Among these miRNAs, 26 and 5 were significantly upregulated and downregulated in the volcano plot, respectively (Figure 1(c), Table 4).

3.2. Target Gene Prediction for Differentially Expressed Exosomal miRNAs. The top ten most upregulated exosomal miRNAs (hsa-miR-129-5p, hsa-miR-130b-3p, hsa-miR2113, hsa-miR-302d-3p, hsa-miR-320b, hsa-miR-320c,
hsa-miR-320d, hsa-miR-320e, hsa-miR-4429, and hsa-miR137-5p) and the top four most downregulated exosomal miRNAs (hsa-let-7e-3p, hsa-miR-223-5p, hsa-miR-501-5p, and hsa-miR-584-5p) were selected for evaluation. Target genes were predicted using TargetScan and miRDB, generating 4640 potential target genes, including 3542 genes for upregulated and 1098 genes for downregulated miRNAs.

3.3. Functional Analysis of Differentially Expressed Exosomal miRNA Target Genes. To further highlight the functional features of exosomal miRNAs, the target genes were annotated using GO terms. The target genes of upregulated miRNAs were mainly enriched in "regulation of nitrogen compound metabolic process" (208 genes, $P=3.14 E-12$ ), "nuclear lumen"(145 genes, $P=6.31 E-10)$, “doublestranded DNA binding" (47 genes, $P=4.34 E-07$ ), and so on (Figures 2(a), 2(c), 2(d), and 2(e), Table S1). The target genes of downregulated miRNAs were mainly enriched in "response to water deprivation" (two genes, $P=6.25 E-04$ ), "dendritic_spine" (four genes, $P=7.63 E-03$ ), "HMG_box_ 
Sig pathway of target genes of upregulated miRNA

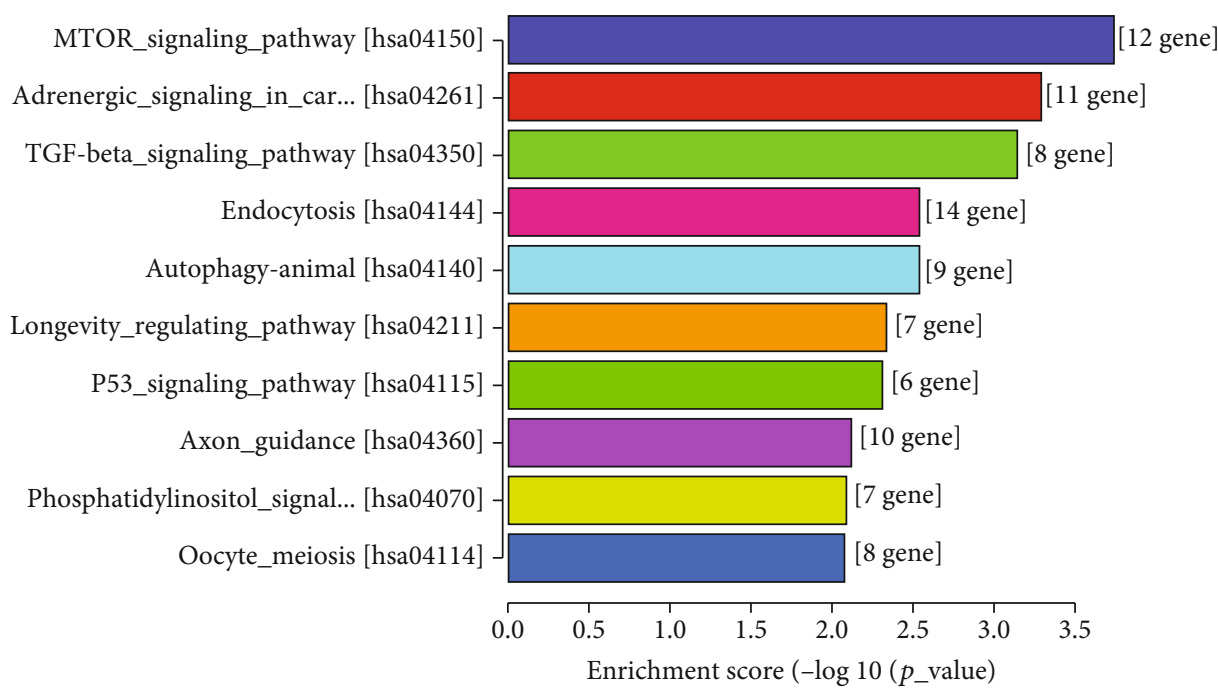

(a)

Sig pathway of target genes of downregulated miRNA

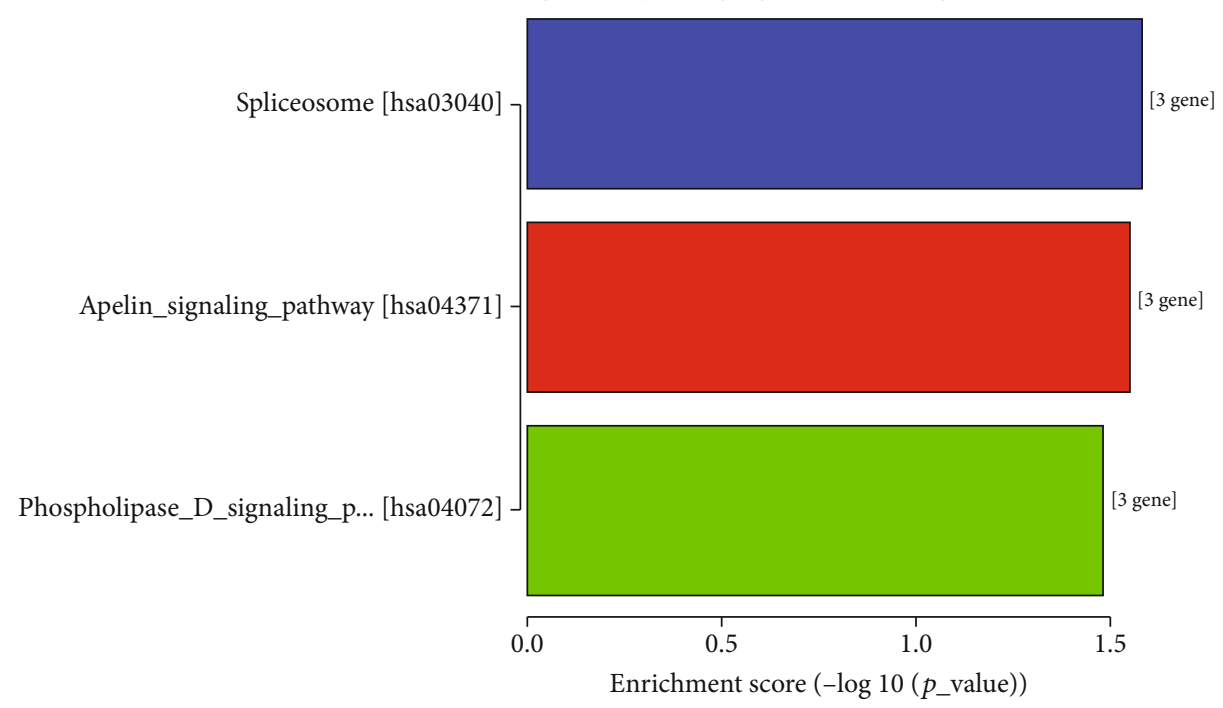

(b)

Figure 3: Continued. 


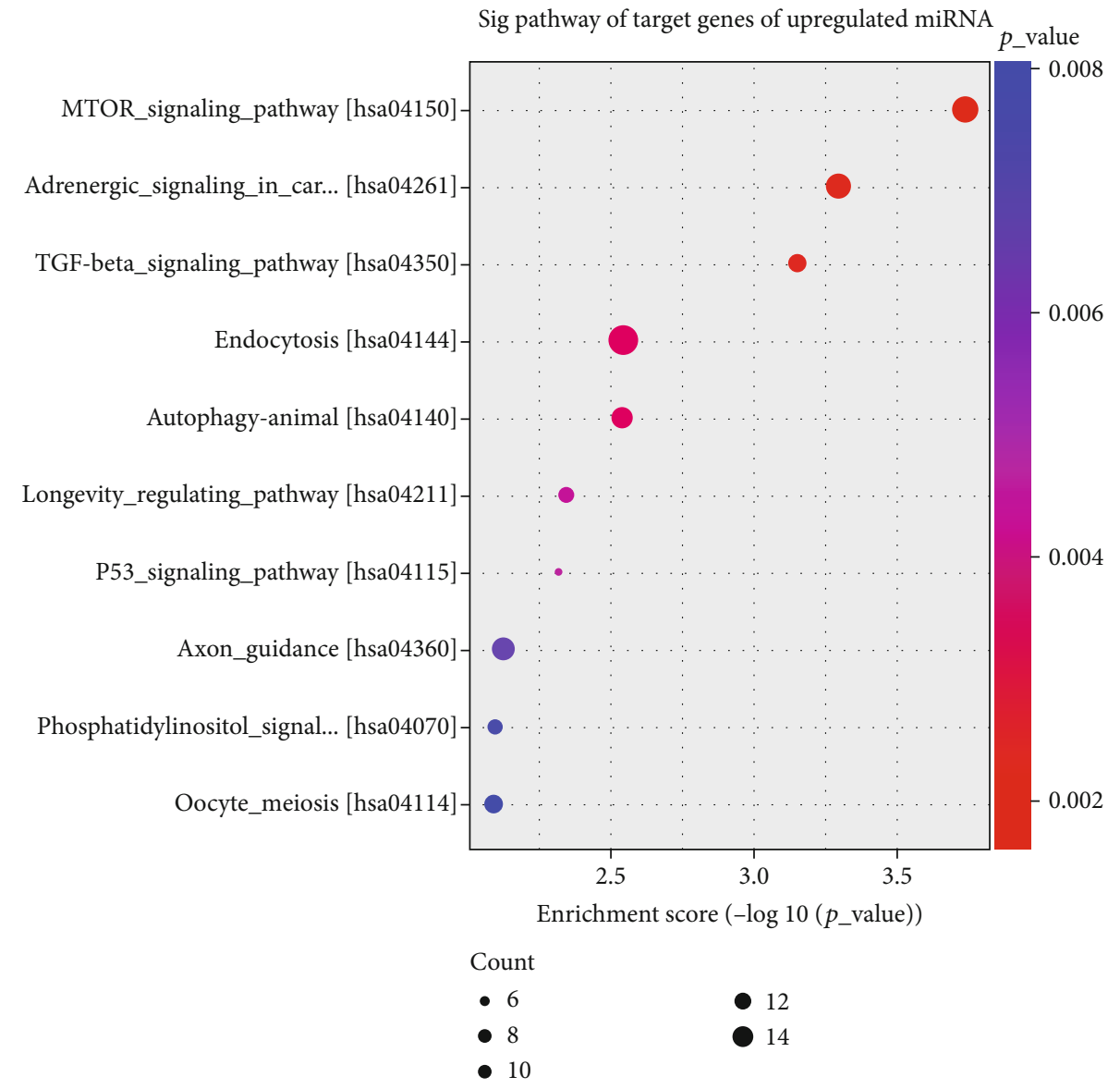

(c)

Figure 3: Continued. 


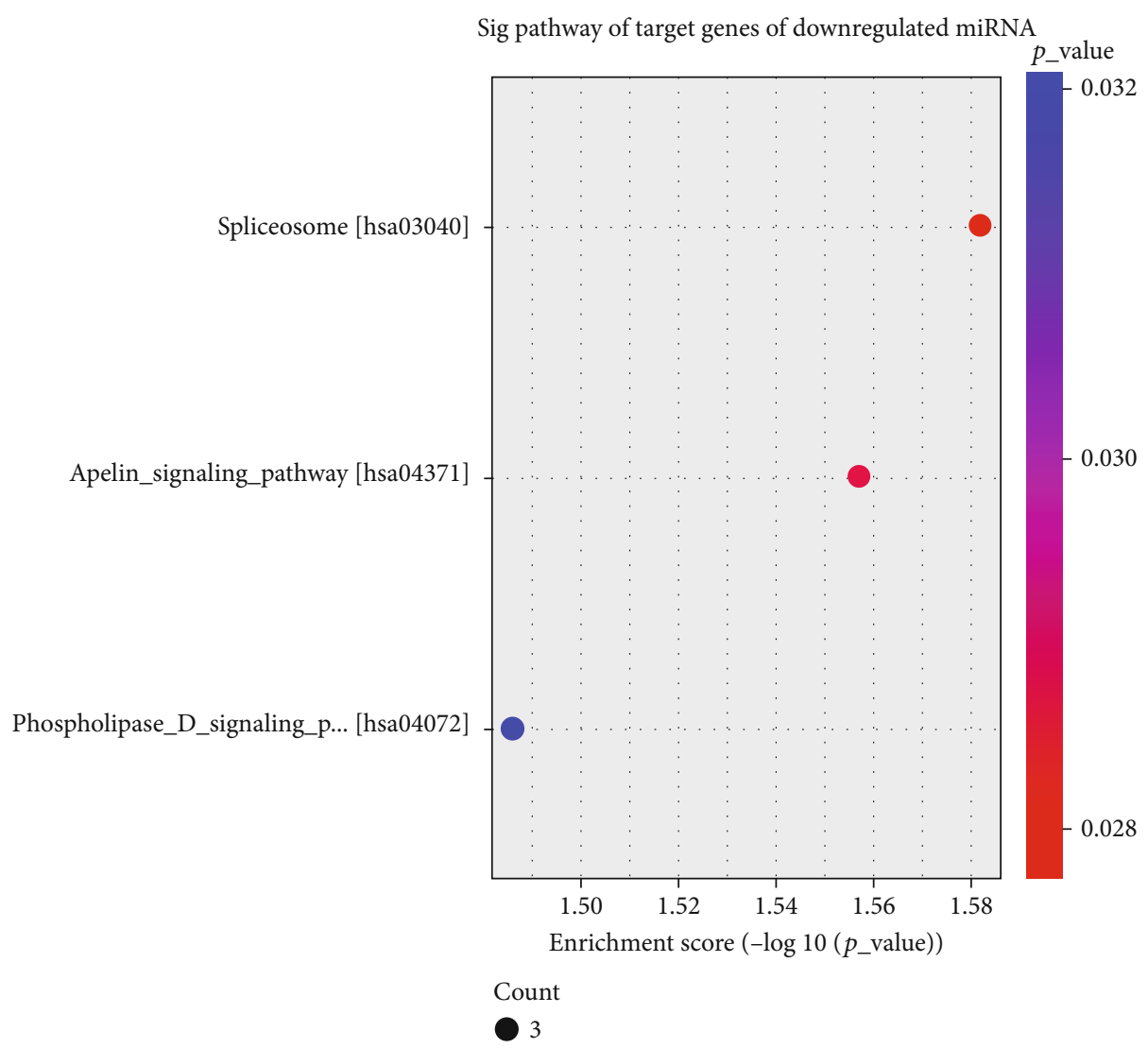

(d)

FIGURE 3: KEGG pathway significantly enriched in the predicted target genes of differentially expressed exosomal miRNAs in CH patients. (a, c) Enriched top 5 pathways of differentially upregulated miRNA target genes in CH patients compared with CS. (b, d) Enriched top 3 pathways of differentially downregulated miRNA target genes in $\mathrm{CH}$ patients compared with CS. Size and color of the bubble represented the amount of differentially expressed genes enriched in the pathway and enrichment significance, respectively.

domain_binding" (two genes, $P=3.18 E-03$ ), and so on (Figures 2(b), 2(f), 2(g), and 2(h), Table S2).

KEGG enrichment analysis demonstrated that target genes were significantly enriched in 54 signaling pathways, of which "mTOR signaling_pathway" (12 genes, $P=1.83 E$ - 04) was the most significantly enriched pathway of upregulated miRNA target genes (Figures 3(a) and 3(c), Table S3), and "spliceosome" (three genes, $P=2.62 E-02$ ) was the most significantly enriched pathway of downregulated miRNA target genes (Figures 3(b) and 3(d), Table S3). These results suggest that $\mathrm{CH}$ has various genetic and phenotypic characteristics.

3.4. Real-Time qPCR Validation of Differentially Expressed Exosomal miRNAs. Among these signaling pathways, we selected PTEN as a target gene in "nervous system development", because this has been related to hydrocephalus in previous reports [17] (Figure 4(a), Figure S1). The miRNA corresponding to PTEN was hsa-miR-130b-3p. In addition, hsa-miR-2113 and hsa-miR-501-5p were noticeably differentially expressed in the $\mathrm{CH}$ patients (Table 4). Future studies should be carried out focusing on larger samples at an individual level. We performed real-time qPCR validation of these three miRNAs in cerebrospinal fluid exosomes from $15 \mathrm{CH}$ patients and $21 \mathrm{CS}$ and revealed that hsa-miR-130b-3p was upregulated, while hsamiR-501-5p was downregulated, in $\mathrm{CH}$ patients compared with CS (Figures 4(b) and 4(c)). However, there was no significant difference in hsa-miR-2113 expression between $\mathrm{CH}$ patients and CS (Figure 4(d)). The trends in expression levels of these two miRNAs according to qPCR were in accordance with the miRNA sequencing results.

3.5. Upregulation of hsa-miR-130b-3p Decreased Expression of PTEN via the Predicted Binding Site. PTEN is a potential target gene of hsa-miR-130b-3p. PTEN was downregulated in the $15 \mathrm{CH}$ patients compared with $21 \mathrm{CS}$, according to the real-time $\mathrm{qPCR}$ results, in contrast to the trend for hsa-miR-130b-3p (Figure 5(a)). The TargetScan showed the predicted binding sites in the $3^{\prime}$-UTR of PTEN with hsa-miR-130b-3p (Figure 5(b)). hsa-miR-130b-3p mimics significantly suppressed luciferase reporter activity compared with NC mimics, after transfection with pGL3-promoter-PTEN-3'-UTR (Figure 5(c)). To avoid unspecific binding, the binding site in pGL3-promoter-PTEN-3'-UTR was deleted. Transfection of hsa-miR-130b-3p mimics significantly inhibited pGL3-promoter-PTEN-3'-UTR-WT 
Sig GO-BP terms of target genes of upregulated miRNA

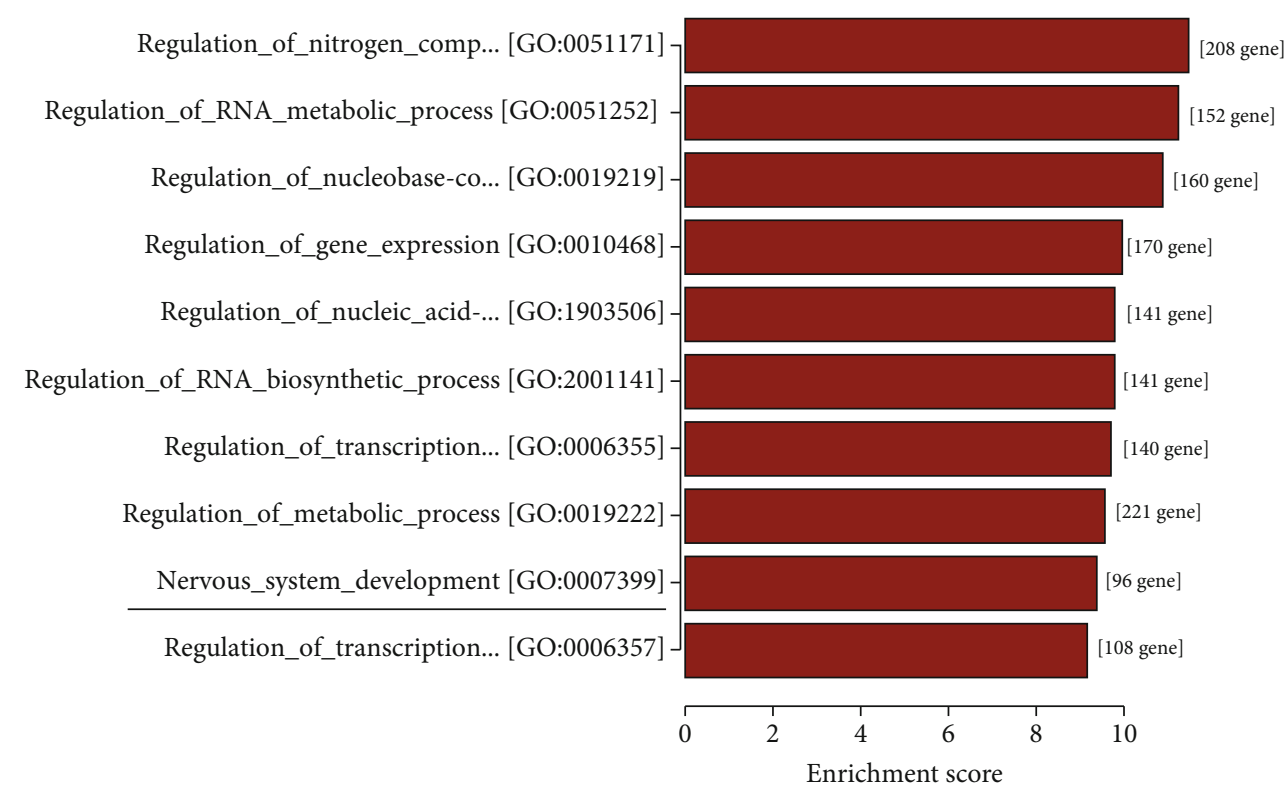

(a)

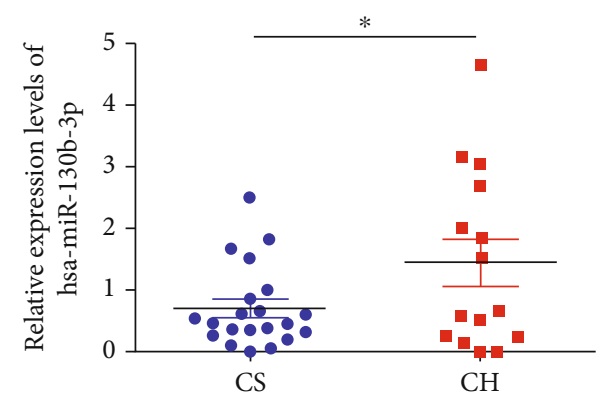

(b)

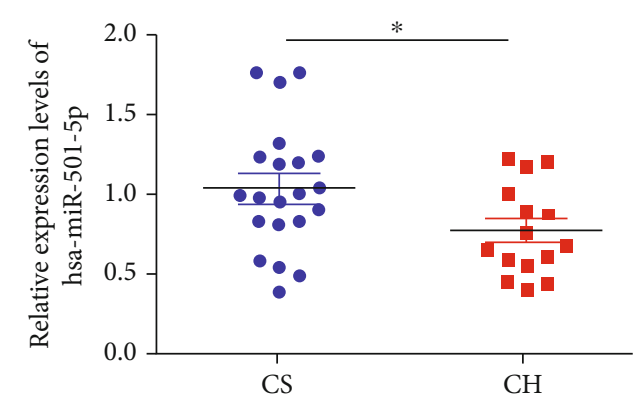

(c)

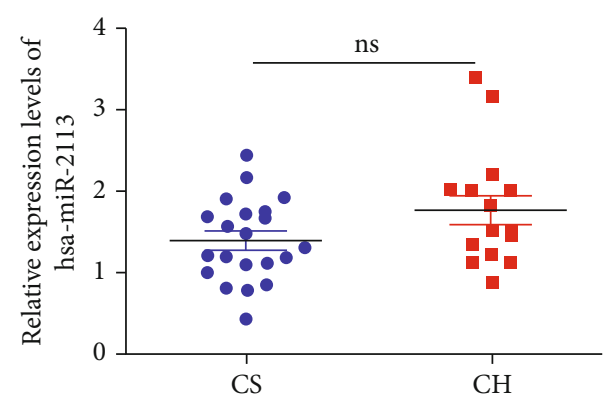

(d)

FIGURE 4: Real-time qPCR validation of differentially expressed exosomal miRNAs. (a) The GO-BP term "nervous system development" indicated by the black line was our concern. (b, c, d) The relative expression levels of hsa-miR-130b-3p, hsa-miR-501-5p, and hsa-miR2113 in $\mathrm{CH}$ patients compared with CS by real-time qPCR. Values are represented as the mean \pm SEM. $n=3 ;{ }^{*} P<0.05$.

activity, but had no effect on pGL3-promoter-PTEN-3' UTR-Del activity (Figure 5(d)). These results show that upregulation of hsa-miR-130b-3p regulated downregulation of PTEN via the predicted binding site.

\section{Discussion}

Extracellular circulating miRNAs exist in most human body fluids, including cerebrospinal fluid, and are highly stable
[18]. The delivery of miRNAs to recipient cells in circulating exosomes provides a novel method of intercellular communication. Dysregulation of exosomal miRNAs is an emerging element in a number of diseases, which reveals the important roles of exosomal miRNAs in both physiological and pathological pathways [9]. Several studies have implicated the function of exosomal miRNAs in some kinds of hydrocephalus. For example, Spaull et al. provided the first evidence for exosomes and exosomal miRNA expression in 


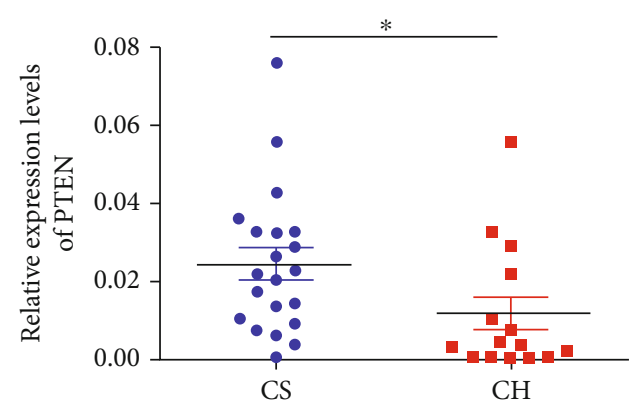

(a)

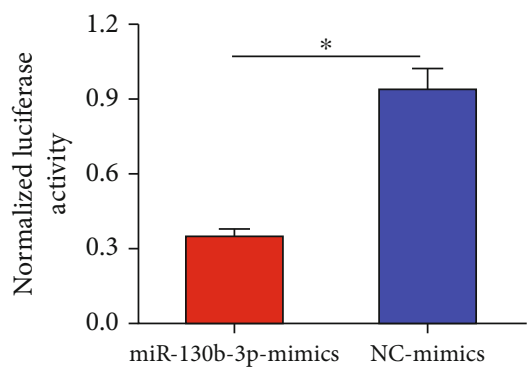

(c)

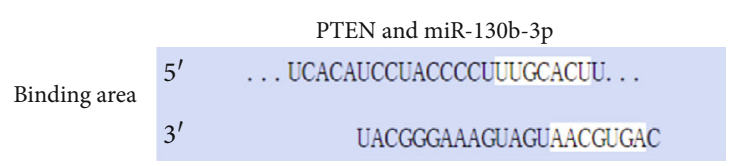

(b)

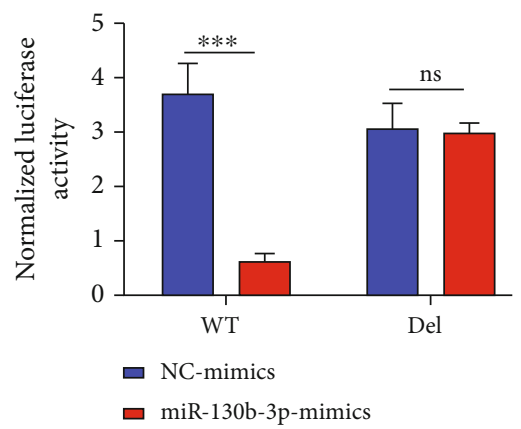

(d)

FIGURE 5: Upregulation of hsa-miR-130b-3p decreased expression of PTEN via the predicted binding site. (a) PTEN was downregulated in the $15 \mathrm{CH}$ patients compared with the $21 \mathrm{CS}$ by qPCR analysis. (b) The predicted binding site of PTEN and hsa-miR-130b-3p. The white area indicates the binding site of PTEN and hsa-miR-130b-3p. (c, d) The normalized luciferase activity of PTEN following transfection with hsa-miR-130b-3p mimics and NC mimics. Values are represented as the mean \pm SEM. $n=3 ;{ }^{*} P<0.05$ and ${ }^{* * *} P<0.001$.

the cerebrospinal fluid in patients with posthemorrhagic hydrocephalus, and the increase in miR-1991-5P following the development of posthemorrhagic hydrocephalus made this an interesting potential biomarker [19]. hsa-miR-4274 was identified as a potential cerebrospinal fluid biomarker for idiopathic normal pressure hydrocephalus, with diagnostic potential, as well as the ability to predict the response to shunt treatment [20]. A prolonged elevation was shown in grade IV vs. grade III of intraventricular hemorrhage with higher miR-155 and miR-181b expression in cerebrospinal fluid at days 41-60 after intraventricular hemorrhage. These alterations may contribute to the development of later clinical complications in this clinical condition [21]. However, the role of exosomal miRNAs in the cerebrospinal fluid of patients with $\mathrm{CH}$ remains unclear. We therefore sequenced exosomal miRNAs in cerebrospinal fluid samples from three $\mathrm{CH}$ patients and three CS to compare the miRNA expression profiles and explore their functions in $\mathrm{CH}$. As it has been reported that age and gender differences affect the expression pattern of miRNA in exosomes, the $\mathrm{CH}$ patients were compared with the age-sex-matched CS in our study [22, 23]. We identified 31 significantly expressed exosomal miRNAs in $\mathrm{CH}$, including 26 that were upregulated and 5 that were downregulated. Among these differentially expressed miRNAs, hsa-miR-2113 and hsa-miR-501-5p were the most significantly upregulated and downregulated, respectively. Previous studies indicated that hsa-miR-2113 was associated with epithelial-mesenchymal transition in diabetes [24] and hepatocellular carcinoma [25], while hsa-miR-501-5p played an important role in modulating tumor progression, e.g., in hepatocellular carcinoma [26], gastric cancer [27], and colorectal cancer [28]. However, their roles in $\mathrm{CH}$ have not been investigated.

Because the main mechanism of miRNA is that it can recognize the target mRNA through base complementary pairing and guide the silencing complex to degrade the target mRNA or block the translation of the target mRNA according to the degree of complementarity, we predicted the target mRNAs of differentially expressed exosomal miRNAs and carried out GO and KEGG pathway enrichment analyses to reveal the biological processes and functions of the target mRNAs. Then, we found hsa-miR-130b-3p was predicted to be combined with $P T E N$, which was enriched in the "nervous system development" pathway. hsa-miR$130 \mathrm{~b}-3 \mathrm{p}$ is known to be involved in cancer progression and various inflammatory diseases [29], and no studies have reported on the association between hsa-miR-130b-3p and $\mathrm{CH}$; PTEN is located on 10q23.3 and encodes a lipid phosphatase with important roles in intracellular signal transduction through dephosphorylation of substrates such as Akt and S6 kinase [30]. Previous studies have suggested that PTEN was required for brain formation, and that dysregulation of PTEN resulted in abnormal brain development and progressive hydrocephalus [17]. A novel germline mutation of the PTEN gene is associated with VATER hydrocephalus syndrome [31]. And hsa-miR-130b-3p has been reported to 
negatively regulate PTEN by binding to the $3^{\prime}$-UTR in PTEN [29-31]. These suggested that hsa-miR-130b-3p was likely to play a pivotal role in the development of $\mathrm{CH}$ by targeting PTEN.

We therefore selected these three miRNAs (hsa-miR2113, hsa-miR-501-5p, and hsa-miR-130b-3p) and PTEN for real-time qPCR validation. Because the cerebrospinal fluid samples were difficult to collect and susceptible to infection, we only collected $15 \mathrm{CH}$ patients and $21 \mathrm{CS}$ for real-time qPCR validation. hsa-miR-130b-3p and hsa-miR501-5p were upregulated and downregulated, respectively, in $\mathrm{CH}$ patients compared with $\mathrm{CS}$, in accordance with the miRNA sequencing results, while there was no significant difference for hsa-miR-2113. miRNA sequencing is a screening method in small samples, and the results of comparison between groups only suggest the possible differences and the false positive results also exist. Furthermore, real-time qPCR showed that PTEN was downregulated in $\mathrm{CH}$ patients, in contrast to the trend for hsa-miR-130b-3p. Dual-luciferase reporter assay showed that hsa-miR-130b-3p regulated the expression of PTEN by binding to the predicted site on the $3^{\prime}$-UTR. Therefore, upregulation of hsa-miR-130b-3p may be involved in the development of $\mathrm{CH}$ via interacting with PTEN and mediating its downregulation.

To the best of our knowledge, this study provides the first report of the expression profiles of exosomal miRNAs in $\mathrm{CH}$. Exosomal hsa-miR-130b-3p and hsa-miR-501-5p may be involved in the development of $\mathrm{CH}$. The mechanism of hsa-miR-130b-3p in $\mathrm{CH}$ has also been partly revealed. These findings will help to provide new diagnostic and therapeutic targets for $\mathrm{CH}$.

\section{Data Availability}

The datasets used and/or analyzed during the current study are available from the corresponding authors on reasonable request.

\section{Ethical Approval}

This study was approved by the Institutional Research Ethics Committee of the Children's Hospital of Fudan University, Shanghai, China (2016-121).

\section{Conflicts of Interest}

The authors declare that there is no conflict of interest regarding the publication of this paper.

\section{Authors' Contributions}

J.M., J.Z., and D.M. were responsible for the idea, project design, and concept of the paper. S.C. performed bioinformatics analysis. S.C., L.H., T.J., and J.Z. collected the clinical samples and information. S.C., P.W., L.H., and B.Z. performed the clinical sample detection and in vitro experiments. J.M., S.C., J.Z., and D.M. wrote, edited, and revised the manuscript. All authors read and approved the manu- script. Shiyu Chen, Hao Li, and Jicui Zheng contributed equally to this work.

\section{Acknowledgments}

This study was supported by grants from the National Key Research and Development Program of China (Nos. 2016YFC1000500 and 2021YFC2701000).

\section{Supplementary Materials}

Supplementary 1. Figure S1: the target gene PTEN in the significant enriched KEGG pathway (mTOR signaling pathway).

Supplementary 2. Table S1: the mainly enriched pathways of target genes of upregulated miRNAs in GO analysis. Table S2: the mainly enriched pathway of target genes of downregulated miRNA in GO analysis. Table S3: the mainly enriched pathways of target genes of dysregulated miRNAs in KEGG analysis.

\section{References}

[1] R. Shaheen, M. A. Sebai, N. Patel et al., "The genetic landscape of familial congenital hydrocephalus," Annals of Neurology, vol. 81, pp. 890-897, 2017.

[2] P. Q. Duy, C. G. Furey, and K. T. Kahle, "Trim71/lin-41 links an ancient miRNA pathway to human congenital hydrocephalus," Trends in Molecular Medicine, vol. 25, pp. 467-469, 2019.

[3] H. M. Tully and W. B. Dobyns, "Infantile hydrocephalus: a review of epidemiology, classification and causes," European Journal of Medical Genetics, vol. 57, pp. 359-368, 2014.

[4] J. Zhang, M. A. Williams, and D. Rigamonti, "Genetics of human hydrocephalus," Journal of Neurology, vol. 253, pp. 1255-1266, 2006.

[5] C. G. Furey, X. Zeng, W. Dong et al., "Human genetics and molecular mechanisms of congenital hydrocephalus," World Neurosurgery, vol. 119, pp. 441-443, 2018.

[6] W. P. Kloosterman and R. H. Plasterk, "The diverse functions of microRNAs in animal development and disease," Developmental Cell, vol. 11, no. 4, pp. 441-450, 2006.

[7] J. Zhang, S. Li, L. Li et al., "Exosome and exosomal microRNA: trafficking, sorting, and function," Genomics, Proteomics \& Bioinformatics, vol. 13, pp. 17-24, 2015.

[8] X. Jin, Y. Chen, H. Chen et al., "Evaluation of tumor-derived exosomal miRNA as potential diagnostic biomarkers for early-stage non-small cell lung cancer using next-generation sequencing," Clinical Cancer Research, vol. 23, pp. 53115319, 2017.

[9] S. K. Ajit, "Circulating microRNAs as biomarkers, therapeutic targets, and signaling molecules," Sensors, vol. 12, pp. 33593369, 2012.

[10] S. Keller, M. P. Sanderson, A. Stoeck, and P. Altevogt, "Exosomes: from biogenesis and secretion to biological function," Immunology Letters, vol. 107, pp. 102-108, 2006.

[11] M. Hosseini, S. Khatamianfar, S. M. Hassanian et al., "Exosome-encapsulated microRNAs as potential circulating biomarkers in colon cancer," Current Pharmaceutical Design, vol. 23, pp. 1705-1709, 2017. 
[12] K. Zhao, G. Liang, X. Sun, and L. L. Guan, "Comparative miRNAome analysis revealed different miRNA expression profiles in bovine sera and exosomes," BMC Genomics, vol. 17, no. 1, p. 630, 2016.

[13] K. Wang, S. Zhang, B. Marzolf et al., "Circulating microRNAs, potential biomarkers for drug-induced liver injury," Proceedings of the National Academy of Sciences of the United States of America, vol. 106, pp. 4402-4407, 2009.

[14] J. J. Chen, B. Zhao, J. Zhao, and S. Li, "Potential roles of exosomal microRNAs as diagnostic biomarkers and therapeutic application in Alzheimer's disease," Neural Plasticity, vol. 2017, Article ID 7027380, 12 pages, 2017.

[15] Y. Gui, H. Liu, L. Zhang, W. Lv, and X. Hu, “Altered microRNA profiles in cerebrospinal fluid exosome in Parkinson disease and Alzheimer disease," Oncotarget, vol. 6, pp. 3704337053, 2015.

[16] Z. Fejes, J. Erdei, M. Pócsi et al., "Elevated pro-inflammatory cell-free microRNA levels in cerebrospinal fluid of premature infants after intraventricular hemorrhage," International Journal of Molecular Sciences, vol. 21, 2020.

[17] A. Ohtoshi, "Hydrocephalus caused by conditional ablation of the Pten or beta-catenin gene," Cerebrospinal Fluid Research, vol. 5, no. 1, p. 16, 2008.

[18] J. A. Weber, D. H. Baxter, S. Zhang et al., "The microRNA spectrum in 12 body fluids," Clinical Chemistry, vol. 56, no. 11, pp. 1733-1741, 2010.

[19] R. Spaull, B. McPherson, A. Gialeli et al., "Exosomes populate the cerebrospinal fluid of preterm infants with posthaemorrhagic hydrocephalus," International Journal of Developmental Neuroscience, vol. 73, pp. 59-65, 2019.

[20] I. Jurjevic, M. Miyajima, I. Ogino et al., "Decreased expression of hsa-miR-4274 in cerebrospinal fluid of normal pressure hydrocephalus mimics with parkinsonian syndromes," Journal of Alzheimer's Disease, vol. 56, pp. 317-325, 2017.

[21] Z. Fejes, M. Pócsi, J. Takai et al., "Preterm intraventricular hemorrhage-induced inflammatory response in human choroid plexus epithelial cells," International Journal of Molecular Sciences, vol. 22, 2021.

[22] E. Prieto-Fernández, E. Lopez-Lopez, I. Martin-Guerrero et al., "Variability in cerebrospinal fluid microRNAs through life," Molecular Neurobiology, vol. 57, pp. 4134-4142, 2020.

[23] E. Prieto-Fernández, A. M. Aransay, F. Royo et al., “A comprehensive study of vesicular and non-vesicular miRNAs from a volume of cerebrospinal fluid compatible with clinical practice," Theranostics, vol. 9, pp. 4567-4579, 2019.

[24] L. P. Xue, X. L. Fu, M. Hu et al., "Rg1 inhibits high glucoseinduced mesenchymal activation and fibrosis via regulating miR-2113/RP11-982M15.8/Zeb1 pathway," Biochemical and Biophysical Research Communications, vol. 501, pp. 827-832, 2018.

[25] L. Zhang, Y. Chen, C. Bao, X. Zhang, and H. Li, "Eukaryotic initiation factor 4AIII facilitates hepatocellular carcinoma cell proliferation, migration, and epithelial-mesenchymal transition process via antagonistically binding to WD repeat domain 66 with miRNA-2113," Journal of Cellular Physiology, vol. 235, no. 11, pp. 8199-8209, 2020.

[26] D. H. Huang, G. Y. Wang, J. W. Zhang, Y. Li, X. C. Zeng, and $\mathrm{N}$. Jiang, "miR-501-5p regulates CYLD expression and promotes cell proliferation in human hepatocellular carcinoma," Japanese Journal of Clinical Oncology, vol. 45, pp. 738-744, 2015.
[27] D. Fan, B. Ren, X. Yang, J. Liu, and Z. Zhang, "Upregulation of miR-501-5p activates the wnt $/ \beta$-catenin signaling pathway and enhances stem cell-like phenotype in gastric cancer," Journal of Experimental \& Clinical Cancer Research: CR, vol. 35, no. 1, p. 177, 2016.

[28] R. Pan, W. Cai, J. Sun, C. Yu, P. Li, and M. Zheng, "Inhibition of KHSRP sensitizes colorectal cancer to 5-fluoruracil through miR-501-5p-mediated ERRFI1 mRNA degradation," Journal of Cellular Physiology, vol. 235, pp. 1576-1587, 2020.

[29] B. Yuan, M. Zou, Y. Zhao, K. Zhang, Y. Sun, and X. Peng, "Upregulation of miR-130b-3p activates the PTEN/PI3K/AKT/ NF- $\kappa \mathrm{B}$ pathway to defense against Mycoplasma gallisepticum (HS strain) infection of chicken," International Journal of Molecular Sciences, vol. 19, no. 8, p. 2172, 2018.

[30] B. D. Manning, "Balancing Akt with S6K: implications for both metabolic diseases and tumorigenesis," The Journal of Cell Biology, vol. 167, pp. 399-403, 2004.

[31] W. Reardon, X. P. Zhou, and C. Eng, "A novel germline mutation of the PTEN gene in a patient with macrocephaly, ventricular dilatation, and features of VATER association," Journal of Medical Genetics, vol. 38, pp. 820-823, 2001. 